\title{
Functional and Radiological Outcomes of Combined Anterior-Posterior Approach Versus Posterior Alone in Management of Isthmic Spondylolisthesis. A Systematic Review and Meta-Analysis
}

\author{
ABDULJABBAR ALHAMMOUD, $\mathrm{MD}^{1}{ }^{1}$ GREGORY SCHROEDER, MD ${ }^{2}$ OSAMA ALDAHAMSHEH, \\ MBBCH,${ }^{1}$ KENAN ALKHALILI, MD ${ }^{3}$ MAYAN LENDNER, BS, ${ }^{2}$ ISAM SAMI MOGHAMIS, MBBS, ${ }^{\prime}$ \\ ALEXANDER R. VACCARO, MD, PHD, MBA ${ }^{2}$ \\ ${ }^{1}$ Hamad Medical Corporation, Doha, Qatar, ${ }^{2}$ Rothman institute, Thomas Jefferson University, Philadelphia, Pennsylvania, ${ }^{3}$ School of Medicine, Cairo University, \\ Cairo, Egypt
}

\begin{abstract}
Background: Lumbar isthmic spondylolisthesis (IS) in adults is defined as the forward slippage of a vertebra onto the top of the vertebra, resulting from a defect in the pars intraarticular, and can be low grade or high grade. Persistent back pain or neurological deficit are indications for surgical intervention. Surgery can be done from back, front, or both, with or without fusion, instrumentation, or decompression, and short or long segment.

Methods: Following the Preferred Reporting Items for Systematic Reviews and Meta-Analyses guidelines, several databases were searched through August 2017 for any observational or experimental studies that evaluated combined anterior-posterior approach versus posterior alone in management of IS. Primary outcome was fusion rate, whereas secondary outcomes included functional outcomes (Visual Analogue Scale [VAS] and Oswestry Disability Index [ODI] score), complication rate (infection, neurological), and reoperation rate. Descriptive, quantitative, and qualitative data were extracted. Most of the cases were low-grade IS.

Results: Of the 645 articles identified, 6 studies were eligible for the meta-analysis, with a total of 397 patients with IS, 198 in the combined (anterior interbody fusion [ALIF] + postero-lateral fusion [PLF]) group and 199 in the posterior (transformational interbody fusion [TLIF]/ postero-lateral interbody fusion [PLIF] + PLF) group, average age of 47.2 years, and 185:212 male : female ratio. Although the fusion rate reached 100\% in some studies, the pooled odds ratio $(\mathrm{OR})$ of fusion rate $(\mathrm{OR}=1.02,95 \%$ confidence interval $[\mathrm{CI}]: 0.294,3.552, P=.972)$ did not reach statistical significance between (ALIF + PLF) versus (TLIF/PLIF + PLF). The estimated pooled standardized mean difference (SMD) showed less blood loss in the anterior approach compared to the posterior approach (SMD $=-0.528,95 \% \mathrm{CI}:-0.777,-0.278, P$ $<.001$ ), with no difference in operative time and length of hospital stay. Despite both groups showing significant improvement in pain and functional scores at final follow up, ODI and VAS were not significantly different between groups with ODI $(\mathrm{SMD}=-0.644,95 \% \mathrm{CI}:-1.948,0.621, P=.311)$ and VAS $(\mathrm{SMD}=0.113,95 \% \mathrm{CI}:-0.173,0.400, P=$ .439). The complication rate for the anterior approach was higher than the posterior, whereas reoperation rate was higher in the posterior approach than the anterior.

Conclusions: No significant difference between anterior and posterior approaches was found in the global assessment of fusion rate and clinical outcomes, despite a higher rate of complications using the anterior approach.

Level of Evidence: 3.

Clinical Relevance: Both anterior and posterior approach are a valid option for treatment of isthemic spondylolisthesis

Lumbar Spine

Keywords: complications, fusion, isthemic, spondylolisthesis
\end{abstract}

\section{INTRODUCTION}

Isthmic spondylolisthesis (IS) is a spine condition that occurs due to the forward slippage of a vertebral body on the underlying one due to a developmental defect in the pars intraarticular (isthmus). The condition is a Type 2 spondylolisthesis according to the Wiltse-Newman classification and can be due to stress fracture, acute fracture, or an elongation defect. ${ }^{1}$ Meyerding classification is a radiological-oriented method of assessing the severity of the slippage in the spondylolisthesis. 
Typically, the opposition occurs in children between 5 and 7 years of age, but the defect persists and can occur in adulthood. The lesion occurs mostly at the level of L5-S1 due to cumulative stress, but can occur above the level of L5 in cases of trauma. In most cases, IS is asymptomatic, but it can present with slow onset lower back pain which can be referred to the buttocks. Other symptoms include tightness of the hamstrings, knee contractures, bladder and bowel incontinence, and rarely cauda equine syndrome. In adolescents, the main concern is instability, whereas in older patients, stenosis is also a main concern.

There are various approaches to the management of IS depending on the severity of the symptoms. Conservative treatment is the mainstay of management, which includes lifestyle modification, thermal therapy, physiotherapy, and nonsteroidal anti-inflammatory drugs. Surgical management of IS is indicated in pain refractory to conservative treatment and interfering with daily activity, the progression of the slip, or development of neurological deterioration. The various surgical techniques described in literature reviews include pars repair, Gill's procedure, ${ }^{2}$ and decompression and fusion of the vertebral bodies with or without instrumentation. Fusion is achieved through different techniques like transformational interbody fusion (TLIF), postero-lateral fusion (PLF), postero-lateral interbody fusion (PLIF), and anterior interbody fusion (ALIF) alone, or with PLF or pedicle screw fixation (PSF).

Different surgical approaches are used to address the condition such as the posterior and anterior approaches. The posterior approach is the initial technique used to address the lesion, allowing for decompression of neural elements and achievement of stability. However, this approach leads to possible worsening of the sagittal alignment and massive injury to paraspinal muscles. The anterior approach, on the other hand, can achieve indirect decompression with possible injuries to surrounding structures. The combined approach has cumulative advantages and disadvantages.

This systematic review aims to present the evidence-based ideal approaches for the surgical management of IS. The review compares the anterior approach (ALIF, ALIF + PLF, ALF + PSF) to the posterior approach (PLF, PLIF, TLIF) in regard to fusion rate, surgical factors (blood loss, surgical time), functional outcomes, complication rates, and radiological changes.

\section{METHODS}

\section{Literature Search}

Relevant comparative studies were identified up to August 2017 following the Preferred Reporting Items for Systematic Reviews and Meta-Analyses guidelines. The literature base was compiled using electronicbased search on MEDLINE (PubMed), EMBASE, and Cochrane databases, and hand searching of abstracts in spine journals (Spine, European Spine Journal, Global Spine Journal, Asian Spine Journal, and Journal of Bone and Joint Surgery).

The following search string was used for search: [(spondylolistheses[Title] OR spondylolisthesis[Title] OR spondylolisthesis[Title] OR spondylolisthesis[Title]) AND ((surgical[Title] AND treatment[Title]) OR fusion[Title] OR (interbody[Title] AND fusion [Title]) OR (posterolateral[Title] AND fusion[Title]) OR (circumferential[Title] AND fusion[Title]) OR (anterior[Title] AND approach[Title]) OR (lateral [Title] AND approach[Title]) OR (posterior[Title] AND approach[Title]) OR TLIF[Title] OR PLIF[Title] OR ALIF[Title] OR DLIF[Title] OR PLF[Title]) AND ("humans"[MeSH Terms] AND English [lang])].

Two investigators independently reviewed all articles that were potentially eligible based on abstract review. Then the eligible studies were selected according to the inclusion and exclusion criteria. Any disagreement was resolved by discussion within the research team.

\section{Study Eligibility Criteria}

The research team systematically reviewed published studies according to the following criteria: (1) subjects were 18 years or older and underwent surgical management for IS; (2) the intervention was done through the anterior approach (ALIF + PLF/ PSF) and the posterior approach (TLIF/PLIF + PLF); (3) the study reported at least 1 desired outcome (fusion rate, functional outcome, surgical parameters, complication rate); (4) patients were followed up at least 1 year after surgery; and (5) studies were excluded if they had reported other types of spondylolisthesis (degenerative, dysplastic, traumatic, or neoplastic).

The primary outcome was fusion rate, whereas the secondary outcomes were (1) functional out- 
comes (Visual Analogue Scale [VAS] pain score, Oswestry Disability Index [ODI] score), (2) complication rate, (3) reoperation rate, (4) surgical parameters (blood loss, blood transfusion, operation time, hospital stay), (5) and radiological changes (disc height, slip angle and slip grade).

\section{Risk of Bias Assessment}

Using the Newcastle-Ottawa Quality Assessment scale, a team of 2 independent coauthors assessed the quality and bias of the publications. This scale assesses the quality of research from 3 points (comparability, selection, and outcome). For the outcome and selection sections, a study earns a maximum of a single star on each of the numbered items on the sections, whereas the study point interbody 2 stars on the upper limit for comparability.

\section{Clinical Relevance}

Two independent reviewers assessed the relevance of the selected studies clinically using the Cochrane Back Review Group recommendations. If a study had some clinical relevance, then it earns a positive recommendation $(+)$, and if not, it receives a negative score $(-)$. On the other hand, an undetermined (?) is given for studies with insufficient data.

\section{Data Collection}

Two independent analyzers conducted the data extraction, with any conflicts being resolved through discussion. The retrieved data included the following:

- The characteristics of the studies (name, year, level of evidence, follow up)

- Subject characteristics (sample size, age, male/ female ratio)

- Disease characteristics (affected level, degree of listhesis)

- Surgical characteristics (number, comparability, fused level, type of graft/cage)

- Primary and secondary outcome parameters as mentioned in the inclusion criteria

\section{Data Analysis}

The data analysis was done by Comprehensive Meta-Analysis software version 3 (Epi Info 2000, Centers for Disease Control and Prevention, Atlanta, Georgia) using a random-effect model and SPSS version 22 (SPSS Inc, Chicago, Illinois). The researcher determined the mean difference or stan-

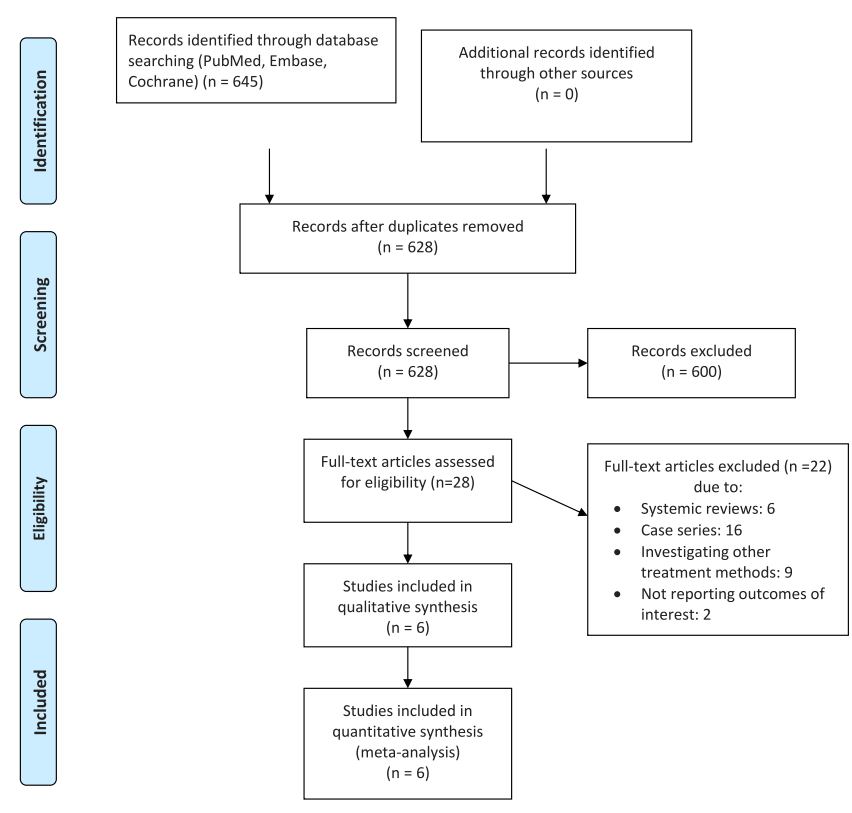

Figure 1. Preferred Reporting Items for Systematic Reviews and MetaAnalyses flow chart.

dardized mean difference (SMD) and 95\% confidence intervals (CIs) for continuous variables, whereas for dichotomous data, analysis was conducted using the odds ratio (OR) and 95\% CI. Statistical heterogeneity across the studies was tested using $\mathrm{I}^{2}$, and the level of evidence was assessed according to the Cochrane Back Review Group.

\section{RESULTS \\ Search Results}

Initial database search revealed 645 studies with 628 studies remaining after removal of duplicates. Six hundred studies were excluded by title and abstract, and 28 full-text studies were reviewed. Out of the 28 studies, only 6 studies met the inclusion criteria, with 22 studies being excluded due to the absence of reported outcomes (Figure 1).

The characteristics of the included studies are reported in Table 1: 6 studies were included involving 397 subjects, 198 in the anterior group and 199 in the posterior group. The average age was 47.2 years, with 185 males and 212 females. The mean follow up was 28.4 months. The most commonly affected level was L5-S1 followed by L4-L5. Most of the cases were low-grade IS.

\section{Risk of Bias Assessment Clinical Relevance}

Reported in Tables 2 and 3. 


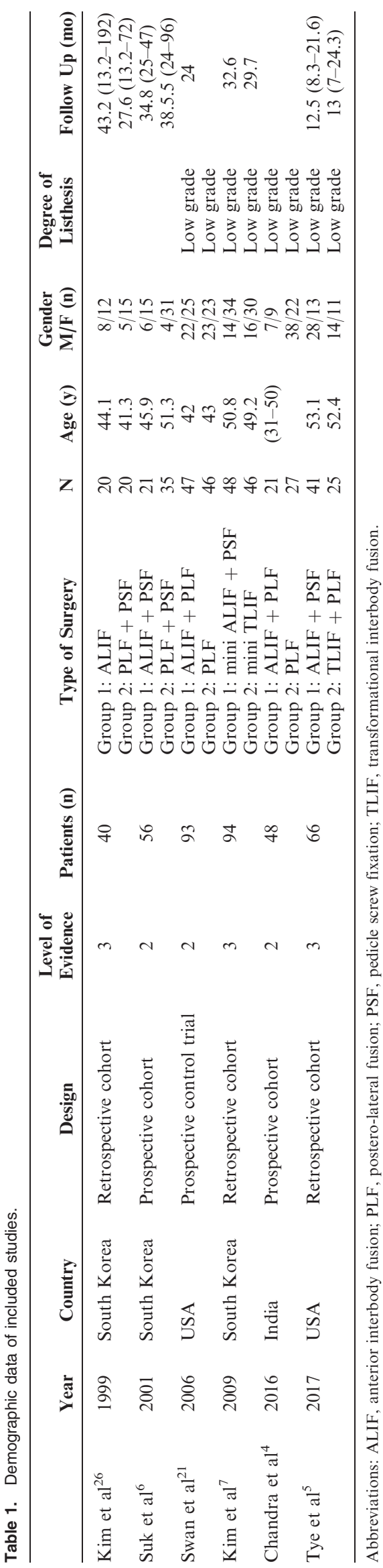

Table 2. Quality assessment. ${ }^{a}$

\begin{tabular}{lccccc}
\hline & Year & $\begin{array}{c}\text { Level of } \\
\text { Evidence }\end{array}$ & Selection & Comparability & Outcome \\
\hline Kim et al $^{26}$ & 1999 & 3 & $* * * *$ & $* *$ & $* * *$ \\
Suk et al $^{6}$ & 2001 & 2 & $* * * *$ & $* *$ & $* * *$ \\
Swan et al $^{21}$ & 2006 & 2 & $* * * *$ & $* *$ & $* * *$ \\
Kim et al $^{7}$ & 2009 & 3 & $* * * *$ & $* *$ & $* * *$ \\
Chandra et al $^{4}$ & 2016 & 2 & $* * * *$ & $* *$ & $* * *$ \\
Tye et al $^{5}$ & 2017 & 3 & $* * * *$ & $* *$ & $* * *$ \\
\hline
\end{tabular}

${ }^{a}$ Asterisks indicate the number of points which each study received in each category of the quality assessment tool.

\section{Meta-Analysis Results}

Most included articles reported high rates of fusion using both approaches, with the estimated pooled $\mathrm{OR}$ of $(\mathrm{OR}=1.02,95 \% \mathrm{CI}: 0.294,3.552, P=$ .972; Figure 2).

Although most of the articles reported longer operative times using the posterior approach, the estimated pooled SMD did not reach statistical significance $(\mathrm{SMD}=0.170,95 \% \mathrm{CI}:-0.591,0.930$, $P=.662$ ). Four studies reported blood loss, with the estimated pooled SMD showing less blood loss using the anterior approach compared to the posterior approach $(\mathrm{SMD}=-0.528,95 \% \mathrm{CI}$ : $-0.777,-0.278, P<.001)$. No statistical difference was detected in length of hospital stay comparing the 2 approaches (SMD $=0.987,95 \% \mathrm{CI}:-0.499$, 2.472, $P=.193$; Figure 3).

Final follow-up ODI score was not significantly different in the anterior and posterior groups (SMD $=-0.644,95 \% \mathrm{CI}:-1.948,0.621, P=.311)$ as well as no significant difference in VAS score at final follow up $(\mathrm{SMD}=0.113,95 \% \mathrm{CI}:-0.173,0.400, P=.439$; Figure 4).

\section{Other Results}

\section{Complication Rate}

The total number of complications in the anterior approach was higher as compared to the posterior

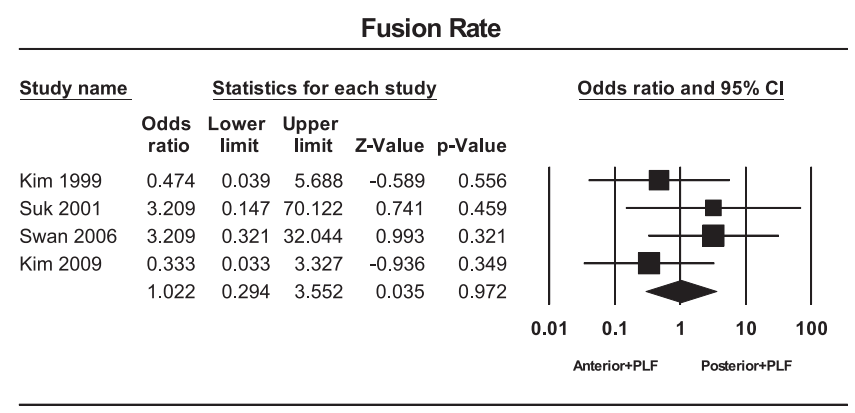

Meta Analysis

Figure 2. Forest plot of fusion rate. 
Table 3. Clinical relevance.

\begin{tabular}{|c|c|c|c|c|c|c|}
\hline Questions & Kim et al $^{26}$ & Suk et al $^{6}$ & Swan et $\mathrm{al}^{21}$ & Kim et $\mathbf{a l}^{7}$ & Chandra et al $^{4}$ & Tye et $\mathrm{al}^{5}$ \\
\hline $\begin{array}{l}\text { 1. Are the patients described in detail so that } \\
\text { you can decide whether they are comparable } \\
\text { to those that you see in your practice? }\end{array}$ & + & + & + & + & + & + \\
\hline $\begin{array}{l}\text { 2. Are the interventions and treatment settings } \\
\text { described well enough so that you can } \\
\text { provide the same for your patients? }\end{array}$ & + & + & + & + & + & + \\
\hline $\begin{array}{l}\text { 3. Were all clinically relevant outcomes } \\
\text { measured and reported? }\end{array}$ & + & + & + & + & + & + \\
\hline 4. Is the size of the effect clinically important? & + & + & + & + & + & + \\
\hline $\begin{array}{l}\text { 5. Are the likely treatment benefits worth the } \\
\text { potential harms? }\end{array}$ & + & + & + & + & + & + \\
\hline
\end{tabular}

one (39 versus 30). Of the complications, transient sensory symptoms were more common in the anterior group, whereas nonunion was more common in the posterior group. Lastly, the reoperation rate was double in the posterior approach comparing to the anterior one (4 versus 8 ; Table 4 ).

\section{Radiological Changes}

All authors reported more improvement in disc height and a greater decrease in slip angle and grade in the ALIF group when compared to posterior procedures alone (PLF and TLIF). Furthermore, ALIF maintained this achievement during final follow up. By far, ALIF leads to improvement in the lumbar lordosis and sagittal balance parameters as compared to TLIF/PLF. However, the correlation between the improvement of radiological parameters and the functional results is still not clear.

\section{DISCUSSION}

This systematic review and meta-analysis identified 6 suitable studies. There is a significant

Table 4. Complication rate.

\begin{tabular}{lcc}
\hline Complication & Anterior & Posterior \\
\hline Position related & 1 & 1 \\
Prolonged ileus & 4 & 0 \\
Other complication & 4 & 2 \\
Urinary tract infection & 5 & 3 \\
Transient sensory symptoms & 5 & 0 \\
Nerve root injury & 1 & 2 \\
Iliac vein laceration & 4 & 0 \\
Transient retrograde ejaculation & 1 & 0 \\
SSI & 4 & 4 \\
Postoperative new-onset clinical depression & 1 & 2 \\
Nonunion & 1 & 3 \\
Delay union & 2 & 0 \\
Hardware failure & 1 & 2 \\
Pseudo-arthrosis & 1 & 1 \\
Reoperation & 4 & 8 \\
Total & 39 & 30 \\
\hline
\end{tabular}

Abbreviation: SSI, surgical site infection. difference in the effectiveness of the anterior and posterior approaches in the management of IS. The discussion is based on the evaluation of radiological fusion and the possible correlation with the clinical outcomes. Despite the broad research studies in the area of IS management, there is still a lot of controversy on the best surgical approach. ${ }^{1,2}$ Surgical management using circumferential, anterior, or the posterior approaches is applied when the conservative management of IS fails. ${ }^{3}$

The posterior approach through PLF/PLIF/ TLIF carries less surgical risks and low costs of operation $^{1-3}$; therefore, it is more popular. Posterolateral fusion has promising results regarding pain relief and improvement in functional outcomes. However, the posterior approach results in less stability of the anterior column and disc height which may lead to postop pain. ${ }^{4-6}$ Due to the poor stability and disc support, there are higher rates of complications. Various studies have been conducted to evaluate the effectiveness of TLIF in the management of IS. Transformational interbody fusion enables the simultaneous decompression of the traversing and exiting nerves and anterior column stabilization. It is also possible to achieve and maintain some lumbar lordosis via TLIF.,8 The approach is made either through an open surgery through a midline lumbar cut or a minimally invasive procedure (MIS) using working tubes and pedicle screws placed percutaneously. The MIS method has less trauma and blood loss but cannot be used for the placement of larger cages.

In contrast, the advantages of ALIF include clear view of the displaced vertebral body, less trauma to the muscles of the back and vertebral ligaments, and less injury to the nerve root. The approach achieves better anterior column stability with less comorbid complications. Anterior interbody fusion is more effective and safe for repair of failed back surgeries. 


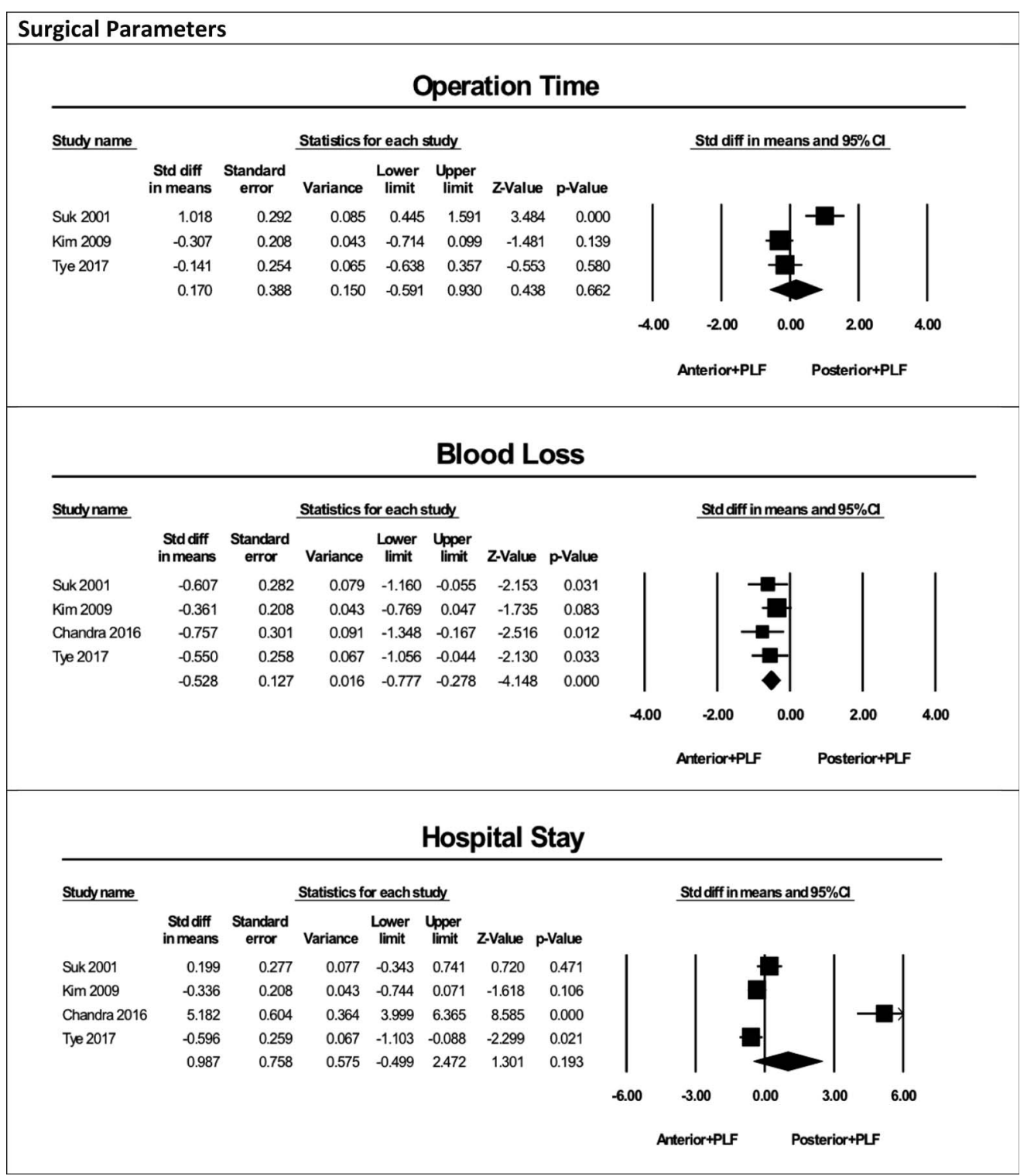

Figure 3. Surgical parameters.

Regarding the fusion rates, Chandra and Singh report a $100 \%$ fusion rate in both ALIF and PLF groups, others reported the superiority of ALIF in fusion rate, and others reported no difference in fusion rate between the anterior and posterior approaches, with superiority in combined approaches. ${ }^{7,9,10}$ A systematic review by Jacobs et $\mathrm{al}^{3}$ could not provide substantial evidence of the superiority of one method over another. A study by Remes et $\mathrm{al}^{11}$ comparing the radiographic and clinical outcomes after circumferential, posterior, and anterior approaches found that the circumferential method results in better outcomes for high-grade IS. ${ }^{3}$ Our meta-analysis failed to find a difference in fusion rate between the anterior and posterior approaches.
The addition of instrumentation to reduce the forward slip still faces a lot of controversy. However, instrumentation has been shown to improve the level of stability and deformity in the posterior approach. ${ }^{5,8,12}$ In other studies, instrumentation leads to increased costs of operation and postsurgical complications coupled with higher blood requirements. ${ }^{5}$

Concerns over adjacent segment degeneration have been raised when comparing anterior versus posterior approaches. In a comparative study, Shim et $\mathrm{al}^{9}$ found that disc degeneration incidence in PLIF is twice that of ALIF. Metal failure is still a concern in instrumentation cases, with more cases reported in PLF managed by salvage ALIF. Other 


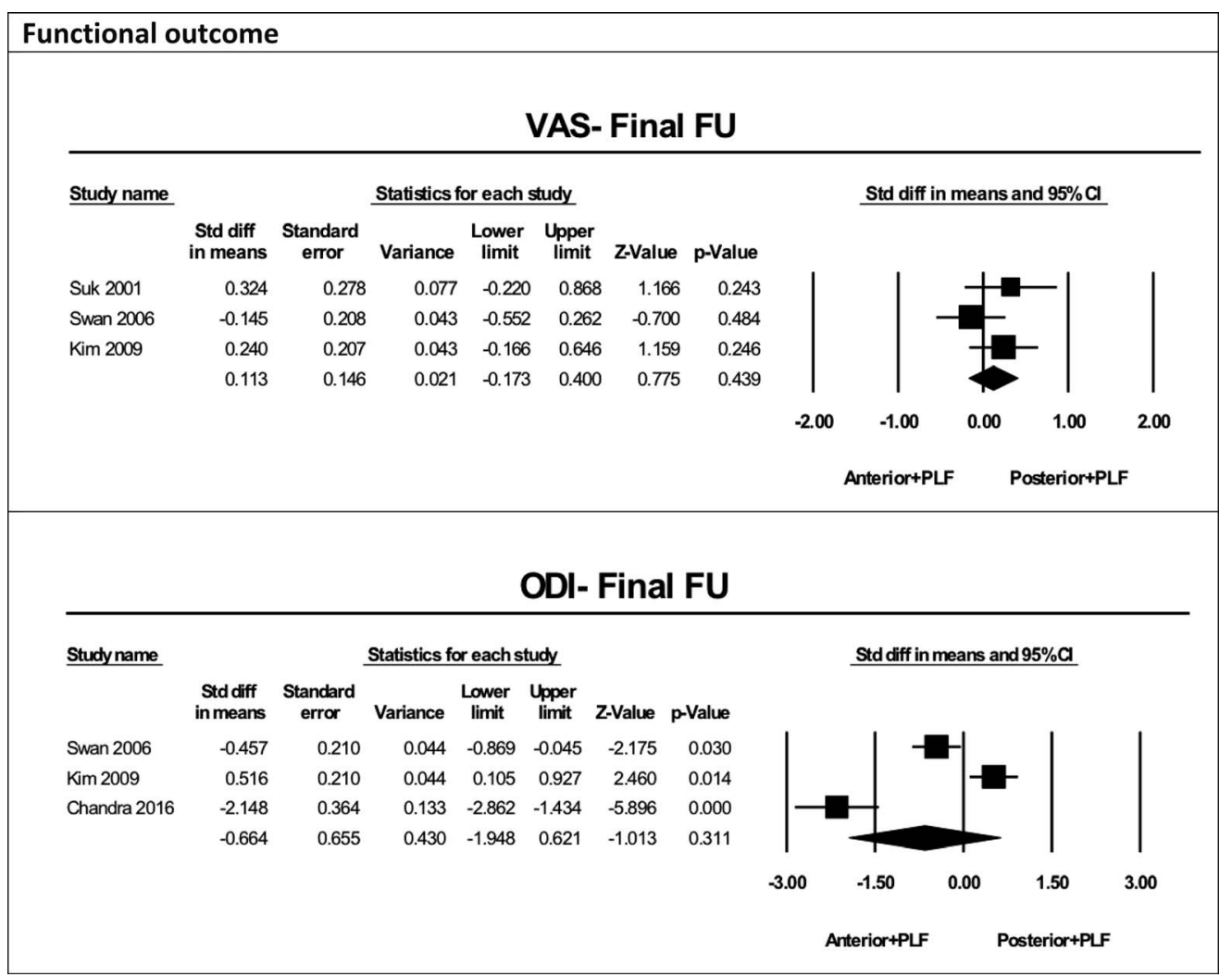

Figure 4. Functional outcome at final follow up.

complications include visceral and vascular complications in $38.3 \%{ }^{4,13}$ Suk et $\mathrm{al}^{6}$ found no major differences in PLF and ALIF regarding rates of complications, relief of pain, and rates of fusion. However, ALIF with instrumentation had better overall outcomes and fewer complications. The total number of complications reported in included studies was higher in the anterior group compared to the posterior group whereas the reoperation rate was double for the posterior approach compared to the anterior approach.

Chandra and Singh's ${ }^{4}$ study reported greater improvement in the functional outcomes of pain and rate of recovery in ALIF as compared to PLF. According to Kim et $\mathrm{al}^{7}{ }^{7}$ both ALIF and TLIF achieved significant improvement in pain between pre-op and postop periods with no difference between the 2 techniques. However, circumferential fusion shows better outcomes in function. ${ }^{14,15}$ Shim et $\mathrm{al}^{9}$ compared the radiological and clinical outcome between ALIF and TLIF in a retrospective analysis; despite the main radiographic improvements in the ALIF as compared to the TLIF adult patients with low-grade IS, there were no significant changes in the clinical and functional results between the 2 cohorts. There was no major difference in the length of recovery period and time to return to normal activity, but ODI measures showed more improvement using TLIF/ALIF at the L4-L5 level. Accordingly, the studies recommend the use of a TLIF approach at the L4-L5 level and an ALIF approach at the L5-S1 level due to the better radiological outcomes for the treatment of unstable IS. ${ }^{4,9,16,17}$ Circumferential fusion shows better outcomes as compared to the posterior approach alone, but there was no substantial difference between the approaches in long-term clinical outcomes and at final follow up.

Postero-lateral fusion requires more surgical exposure and wider incisions up to the level of transverse processes, leading to more blood loss. On the other hand, when the posterior approach exposes tissue only up to the facets, there is less blood loss. ${ }^{18,19}$ Anterior interbody fusion is associated with more complications to the viscera and vasculature. According to Chandra and Singh, ${ }^{4}$ all PLF patients needed a blood transfusion at some point during the treatment, but only 3 patients from the ALIF cohort needed a transfusion. Our metaanalysis found that blood loss is less in anterior approaches as compared to posterior approaches. 
Hospital stay is longer in anterior approaches because the anterior approach ALIF + PLF/PSF is sometimes done in 2 stages. Chandra and Singh $^{4}$ found that the length of hospital stay in the ALIF group was double that of the PLF group, whereas our results did not show any differences in length of hospital stay between anterior and posterior approaches. No difference in operative time was found in anterior versus posterior approaches. However, most of the studies reported less time in ALIF. 7,9,20 Kwon et $\mathrm{al}^{8}$ reported ALIF needed longer operative time, leading to increased morbidity due to more technical difficulties.

Both groups showed some decrease in the slip angle and deformity with the restoration of disc height. However, in posterior fusion alone without instrumentation, the correction of slip angle was not achieved. The posterior approach group shows deterioration of the correction with time on follow-up radiographs, accounting for the increased symptoms. ${ }^{21,22}$ Suk et $\mathrm{al}^{6}$ found that ALIF is better in the prevention of reduction loss. The repair of the slip angle leads to apposition of the central body axis over the sacrum, which leads to decreased tension on the graft. Thus, there is a correction of the axial loading across lumbosacral interspace.

Both approaches lead to improvement of the disc height, especially with big cages. Better results are seen in ALIF as opposed to TLIF/PLF, with ALIF maintaining excellent stability in the first 6 months following surgery until fusion is achieved. Anterior interbody fusion was far better than TLIF/PLF in the restoration of the lumbar lordosis and sagittal balance. ${ }^{17,23,24}$ However, the correlation between the improvement of radiological parameters and functional outcome is still unclear. Goldstein et al found that the changes in lordosis angles after cage and fusion are not good predictors of clinical outcome. $^{7}$ Subsequently, Shim et $\mathrm{al}^{9}$ found that better radiological outcomes did not reflect better clinical outcomes due to short follow up. The maintenance of the anatomical orientation leads to better long-term outcomes due to correction of balance and decreasing the segment stressor. ${ }^{25}$

Cost analysis takes into consideration the cost of the procedure, postsurgical hospital stay cost, follow-up cost, and the cost of complications. Chandra and $\mathrm{Singh}^{4}$ found that the cost of ALIF is higher than PLF due to the complexity of ALIF, instrumentation cost, and cost of hospital stay.
There are some limitations to this systematic review. First, few studies met the eligibility criteria and had good methodological quality. Second, the patient population in the studies is heterogeneous with different levels of disease, which might have some effect on the outcomes of the study. Lastly, the complication and fusion rate criteria in the different studies vary, leading to inconsistent results. Due to the limitations and variance in the methodological approaches to the studies, there may be a high level of bias in outcomes. Therefore, the preferred method of surgical approach to IS may show some variance in different studies.

\section{CONCLUSIONS}

No significant difference between anterior and posterior approaches was found in the global assessment of fusion rate and clinical outcomes, despite a higher rate of complications using the anterior approach.

\section{REFERENCES}

1. Agabegi SS, Fischgrund JS. Contemporary management of isthmic spondylolisthesis: pediatric and adult. Spine $J$. 2010;10(6):530-543.

2. Karampalis C, Grevitt M, Shafafy M, Webb J. Highgrade spondylolisthesis: gradual reduction using Magerl's external fixator followed by circumferential fusion technique and long-term results. Eur Spine J. 2012;21(2):200-206.

3. Jacobs W, Vreeling A, De Kleuver M. Fusion for lowgrade adult isthmic spondylolisthesis: a systematic review of the literature. Eur Spine J. 2005;15(4):391-402. https://doi.org/10. 1007/s00586-005-1021-4.

4. Chandra V, Singh RK. Anterior lumbar interbody fusion with instrumentation compared with posterolateral fusion for low-grade isthmic-spondylolisthesis. Acta Ortho Belg. 2016;82(1):23-30.

5. Tye E, Tanenbaum J, Alonso A, et al. Circumferential fusion: a comparative analysis between anterior lumbar interbody fusion with posterior pedicle screw fixation and transforaminal lumbar interbody fusion for 15-s1 isthmic spondylolisthesis. Spine J. 2018;18(3):464-471. https://doi.org/ 10.1016/j.spinee.2017.08.227

6. Suk K, Jeon C, Park M, Moon S, Kim N, Lee H. Comparison between posterolateral fusion with pedicle screw fixation and anterior interbody fusion with pedicle screws fixation in adult spondylolytic spondylolisthesis. Yonsei Med J. 2001;42(3):316-323.

7. Kim J, Kang B, Lee S, et al. Mini-transforaminal lumbar interbody fusion versus anterior lumbar interbody fusion augmented by percutaneous pedicle screw fixation. $J$ Spinal Disord Tech. 2009;22(2):114-121.

8. Kwon B, Hilibrand A, Malloy K, et al. A critical analysis of the literature regarding surgical approach and outcome for 
adult low-grade isthmic spondylolisthesis. $J$ Spinal Disord Tech. 2005;18(Suppl 1):S30-S40.

9. Shim JH, Kim WS, Kim JH, Kim DH, Hwang JH, Park CK. Comparison of instrumented posterolateral fusion versus percutaneous pedicle screw fixation combined with anterior lumbar interbody fusion in elderly patients with L5-S1 isthmic spondylolisthesis and foraminal stenosis. J Neurosurg Spine. 2011;15(3):311-319.

10. Müslüman A, Yılmaz A, Cansever T, et al. Posterior lumbar interbody fusion versus posterolateral fusion with instrumentation in the treatment of low-grade isthmic spondylolisthesis: midterm clinical outcomes. Spine (Phila Pa 1976). 2011;14(4):488-496.

11. Remes V, Lamberg T, Tervahartiala P, et al. Long-term outcome after posterolateral, anterior, and circumferential fusion for high-grade isthmic spondylolisthesis in children and adolescents. Spine (Phila Pa 1976). 2006;31(21):2491-2499.

12. Habib H. Posterolateral fusion versus posterior interbody fusion in adult lumbar isthmic spondylolisthesis. Menoufia Med J. 2014;27(1):191-196.

13. Ekman P, Möller H, Hedlund R. Predictive factors for the outcome of fusion in adult isthmic spondylolisthesis. Spine (Phila Pa 1976). 2009;34(11):1204-1210.

14. Wang S, Han Y, Liu X, et al. Fusion techniques for adult isthmic spondylolisthesis: a systematic review. Arch Orthop Trauma Surg. 2014;134(6):777-784.

15. Sakaura H, Miwa T, Yamashita T, Kuroda Y, Ohwada T. Posterior lumbar interbody fusion with cortical bone trajectory screw fixation versus posterior lumbar interbody fusion using traditional pedicle screw fixation for degenerative lumbar spondylolisthesis: a comparative study. J Neurosurg Spine. 2016;25(5):591-595.

16. Liu X, Wang Y, Qiu G, Weng X, Yu B. Meta-analysis of circumferential fusion versus posterolateral fusion in lumbar spondylolisthesis. J Spinal Disord Tech. 2014;27(8):E282-E293.

17. Singh K. Treatment of high-grade isthmic spondylolisthesis by anterior lumbar interbody fusion with structural fibular allograft. J Minimal Invas Orthop. 2016;0(5):38-46.

18. Liu X, Qiu G, Weng X, Yu B, Wang Y. What is the optimum fusion technique for adult spondylolisthesis - PLIF or PLF or PLIF Plus PLF? A meta-analysis from 17 comparative studies. Spine (Phila Pa 1976). 2014;39(22):1887-1898.

19. Sakaura H, Miwa T, Yamashita T, Kuroda Y, Ohwada T. Posterior lumbar interbody fusion with cortical bone trajectory screw fixation versus posterior lumbar interbody fusion using traditional pedicle screw fixation for degenerative lumbar spondylolisthesis: a comparative study. J Neurosurg Spine. 2016;25(5):591-595.

20. Hyun Oh C. Mini-open and conventional-open transforaminal lumbar interbody fusion augmented by pedicle screw fixation: comparisonal result of clinical, perioperative parametric, functional and radiological assessments. J Spine. 2013;2(3).

21. Swan J, Carragee E, Malek F, et al. Surgical treatment for unstable low-grade isthmic spondylolisthesis in adults: a prospective controlled study of posterior instrumented fusion compared with combined anterior-posterior fusion. Spine J. 2006;6(6):606-614.

22. El Abed K. The surgical management of low-grade single level adult degenerative spondylolisthesis: posterolateral fusion versus posterior lumbar interbody fusion. MOJ Orthop Rheumatol. 2014;1(1).

23. Xiuxin H, Yue Z, Cui C, Yajun W. A meta-analysis of circumferential fusion versus instrumented posterolateral fusion in the lumbar spine. Spine (Phila Pa 1976). 2009;34(17):E618E625.

24. Jones TR, Rao RD. Adult isthmic spondylolisthesis. $J$ Am Acad Orthop Surg. 2009;17(10):609-617.

25. Dhall SS, Wang MY, Mummaneni PV. Clinical and radiographic comparison of mini-open transforaminal lumbar interbody fusion with open transforaminal lumbar interbody fusion in 42 patients with long-term follow-up. J Neurosurg Spine. 2008;9(6):560-565.

26. Kim NH, Lee JW. Anterior interbody fusion versus posterolateral fusion with transpedicular fixation for isthmic spondylolisthesis in adult. Spine (Phila Pa 1976). 1999;24(8):812-817.

\section{Disclosures and COI: None.}

Corresponding Author: Abduljabbar Alhammoud, MD, Hamad Medical Corporation, P.O. Box 3050, Doha, Qatar. Phone: 0097433273574; Email: Aghammoud85@hotmail.com.

Published 30 June 2019

This manuscript is generously published free of charge by ISASS, the International Society for the Advancement of Spine Surgery. Copyright (c) 2019 ISASS. To see more or order reprints or permissions, see http://ijssurgery.com. 